\title{
Comparative Analysis of SWIRM Domain-Containing Proteins in Plants
}

\author{
Yan Gao, ${ }^{1,2}$ Songguang Yang, ${ }^{1}$ Lianyu Yuan, ${ }^{1,2}$ \\ Yuhai Cui, ${ }^{3}$ and Keqiang $\mathrm{Wu}^{4}$ \\ ${ }^{1}$ Key Laboratory of Plant Resources Conservation and Sustainable Utilization, South China Botanical Garden, \\ Chinese Academy of Sciences, Xingke Road 723, Guangzhou 51065, China \\ ${ }^{2}$ Graduate University of Chinese Academy of Sciences, Chinese Academy of Sciences, Beijing 100049, China \\ ${ }^{3}$ Agriculture and Agri-Food Canada, Southern Crop Protection and Food Research Centre, London, ON, Canada N5V 4T3 \\ ${ }^{4}$ Institute of Plant Biology, National Taiwan University, Taipei 106, Taiwan
}

Correspondence should be addressed to Keqiang Wu, kewu01@gmail.com

Received 12 April 2012; Revised 16 June 2012; Accepted 24 June 2012

Academic Editor: Elena Pasyukova

Copyright (C) 2012 Yan Gao et al. This is an open access article distributed under the Creative Commons Attribution License, which permits unrestricted use, distribution, and reproduction in any medium, provided the original work is properly cited.

\begin{abstract}
Chromatin-remodeling complexes affect gene expression by using the energy of ATP hydrolysis to locally disrupt or alter the association of histones with DNA. SWIRM (Swi3p, Rsc8p, and Moira) domain is an alpha-helical domain of about 85 residues in chromosomal proteins. SWIRM domain-containing proteins make up large multisubunit complexes by interacting with other chromatin modification factors and may have an important function in plants. However, little is known about SWIRM domain-containing proteins in plants. In this study, 67 SWIRM domain-containing proteins from 6 plant species were identified and analyzed. Plant SWIRM domain proteins can be divided into three distinct types: Swi-type, LSD1-type, and Ada2-type. Generally, the SWIRM domain forms a helix-turn-helix motif commonly found in DNA-binding proteins. The genes encoding SWIRM domain proteins in Oryza sativa are widely expressed, especially in pistils. In addition, OsCHB701 and OsHDMA701 were downregulated by cold stress, whereas OsHDMA701 and OsHDMA702 were significantly induced by heat stress. These observations indicate that SWIRM domain proteins may play an essential role in plant development and plant responses to environmental stress.
\end{abstract}

\section{Introduction}

In eukaryotes, the genetic information encoded by DNA is packaged into chromatin. The reversibly dynamic changes in chromatin structure modulate the access of regulatory factors to DNA $[1,2]$. The precise coordination and organization of chromatin modifications are essential for the correct spatial and temporal maintenance of the epigenetic code within the eukaryotic genome $[3,4]$. These changes in chromatin involve activities of many chromatin-modifying complexes, consisting of both catalytic and noncatalytic subunits [5]. Such subunits are characterized by specific structural frames that mediate protein-protein and proteinDNA interactions. Generally these specific function domains are conserved through evolution. At present, some of these conserved chromosomal protein modules are well studied, such as the bromodomain, the chromodomain, and the
SANT domain. Bromodomains were discovered to function as acetyl-lysine binding domains [6, 7]. Chromodomains were commonly found in proteins associated with the remodeling and manipulation of chromatin, mediating specific interactions with proteins and RNA by recognizing lysine methylation in histone tails $[8,9]$. SANT domains tether to both DNA and proteins and are essential for histone acetyltransferase activity $[10,11]$. Another such conserved domain is the SWIRM identified in several remodeling and modifying complexes.

SWIRM domain was named after the proteins Swi3p, Rsc8p, and Moira, in which it was first recognized. The computational sequence-profile analysis indicates that the typical SWIRM domain consists of 85 amino acid residues and forms a compact helix-turn-helix (HTH)-related structure [12]. Based on the domain architectures and the amino acid sequence homology, the SWIRM domains 
can be classified into three main types: Swi3/MYSM1 (human MYb-like, Swirm, and Mpn domain-containing protein-1), LSD1 (Lysine-specific demethylase 1), and Ada2 (Adenosine deaminase isoenzymes 2) types [13]. Swi3p-type SWIRM domain-containing proteins are homologous to the ATP-dependent chromatin remodeling complexes SWI/SNF. LSD1-type SWIRM domain-containing proteins belong to Lysine-specific demethylase. LSD1 is the first histone demethylase discovered, and it belongs to the superfamily of the flavin adenine dinucleotide (FAD)-dependent amine oxidases $[14,15]$. Ada2-types SWIRM domain-containing proteins are homologs of transcriptional adaptor ADA2a, which promotes histone lysine acetylation and transcriptional activation and acts as a molecular scaffold within the SAGA remodeling complex [16].

Recent studies indicate that plant SWIRM domaincontaining proteins function in various plant physiological and developmental processes. In Arabidopsis, there are four variants of Swi3-type proteins. AtSWI3A and AtSWI3B are essential for early embryonic development, whereas AtSWI3C and AtSWI3D affect different phases of vegetative and reproductive development [17]. The swi3b mutants display a reduced sensitivity to $A B A-$ mediated inhibition of seed germination and growth and reduced expression of the ABAresponsive genes [18]. Furthermore, AtSWI3B can interact with FCA, a regulator of flowering time in Arabidopsis [19]. AtSWI3C is a core subunit of a BRM ATPase-associated SWI/SNF complex [20]. In addition, the LSD1-type SWIRM domain-containing protein AtFLD is involved in the floral transition and regulates the reproductive competence of the shoot $[21,22]$.

Although these studies have provided important insights about SWIRM domain-containing proteins in Arabidopsis thaliana, the knowledge on their functions is still scarce in the other plants. In this study, we identified 67 SWIRM domaincontaining proteins from 6 plant species including Arabidopsis thaliana, Medicago truncatula, Oryza sativa, Physcomitrella patens, Populus trichocarpa, and Zea mays. The SWIRM proteins from Oryza sativa were further characterized and their expression patterns were analyzed.

\section{Materials and Methods}

2.1. Identification of the SWIRM Family Proteins in Plants. To identify the SWIRM family proteins in plants, BLAST [23] searches of the NCBI databases (http://www.ncbi.nlm.nih .gov/) were performed using the amino acid sequence of the SWIRM domain in AtSWI3A as a query sequence. All predicted SWIRM domain-containing proteins were used for similarity searches again to confirm these predicted proteins and detect new candidates. The following databases were used in this search: TAIR (The Arabidopsis Information Resource, http://www.arabidopsis .org/), RAPDB (Rice Genome Annotation Project Database and Resource, http://rice.plantbiology.msu.edu/), ProFITs of maize (http://bioinfo.cau.edu.cn/ProFITS/index.php/), and Moss Genome (http://www.mossgenome.org/). We obtained those sequences whose $E$ values were below $1 e^{-5}$ and redundant sequences with different identification numbers and the same chromosome loci were removed from our dataset. Amino acid sequences of SWIRM domain-containing proteins were obtained from NCBI and reconfirmed using the Chromatin Database (http://www.chromdb.org/) and respective databases (TAIR, TIGR, RAPDB, and ProFITs of maize).

\subsection{Phylogenetic Analysis and Multiple Sequence Alignment.} Phylogenetic analysis was performed with the MEGA 4.0 program [24] by the neighbor-joining method. Bootstrap analysis was carried out with 1000 replicates based on the complete amino acid sequences. Amino acid sequences of SWIRM domain in fasta formats were used for multiple sequence alignment by ClustalX [25] and then adjusted manually using the GeneDoc software [26].

2.3. Bioinformatics Analyses. Conserved domains of the SWIRM family proteins were explored by using the following databases: Pfam (http://pfam.janelia.org/), SMART (http:// smart.embl-heidelberg.de/), and CDD (http://www.ncbi .nlm.nih.gov/Structure/cdd/cdd.shtml). The protein secondary structure was predicted by Jpred (http://www.compbio .dundee.ac.uk/www-jpred/) and PSIPRED (http://bioinf.cs .ucl.ac.uk/psipred/). The protein conserved domains were draw by DOG2.0 software [27].

2.4. Expression Analysis of OsSWIRMs. The expression patterns of all SWIRM domain-containing proteins were individually queried using Genevestigator (https://www.genevestigator.com/gv/plant.jsp). The expression levels of OsSWIRMs in the developmental stages, different anatomical parts and abiotic stress were analyzed.

\section{Results}

3.1. Phylogenetic Analyses of SWIRM Domain-Containing Proteins in Plant. The amino acid sequence of the AtSWI3A SWIRM domain was used as a query sequence to perform independent searches in NCBI database, then 67 SWIRM domain-containing proteins were identified from Physcomitrella patens (lower plant), Oryza sativa L. ssp. Japonica and Zea mays (monocot), Medicago truncatula and Arabidopsis thaliana (dicot), and Populus trichocarpa (xylophyta) (Table 1). To further investigate the evolutionary history of SWIRM domain-containing proteins in plants, we carried out phylogenetic analyses with the 67 amino acid sequences using MEGA 4.0 program. The phylogenetic tree (Figure 1) indicates that the 67 SWIRM domain-containing proteins fall into three clades: Swi3-type subfamily, LSD1type subfamily, and Ada2-type subfamily, nevertheless, MYSM1-type SWIRM-containing proteins were not found in plants. Considering the structural resemblance between the Swi3 and LSD1 SWIRM domains, it is possible that the Swi3-type and Ada2-type SWIRM proteins had diverged after the appearance of the LSD1-type SWIRM. The plant SWI3 homologues can be clearly divided into four groups: SWI3A, SWI3B, SWI3C, and SWI3D, based on four Arabidopsis variants. There are six Swi3-type SWIRM proteins 


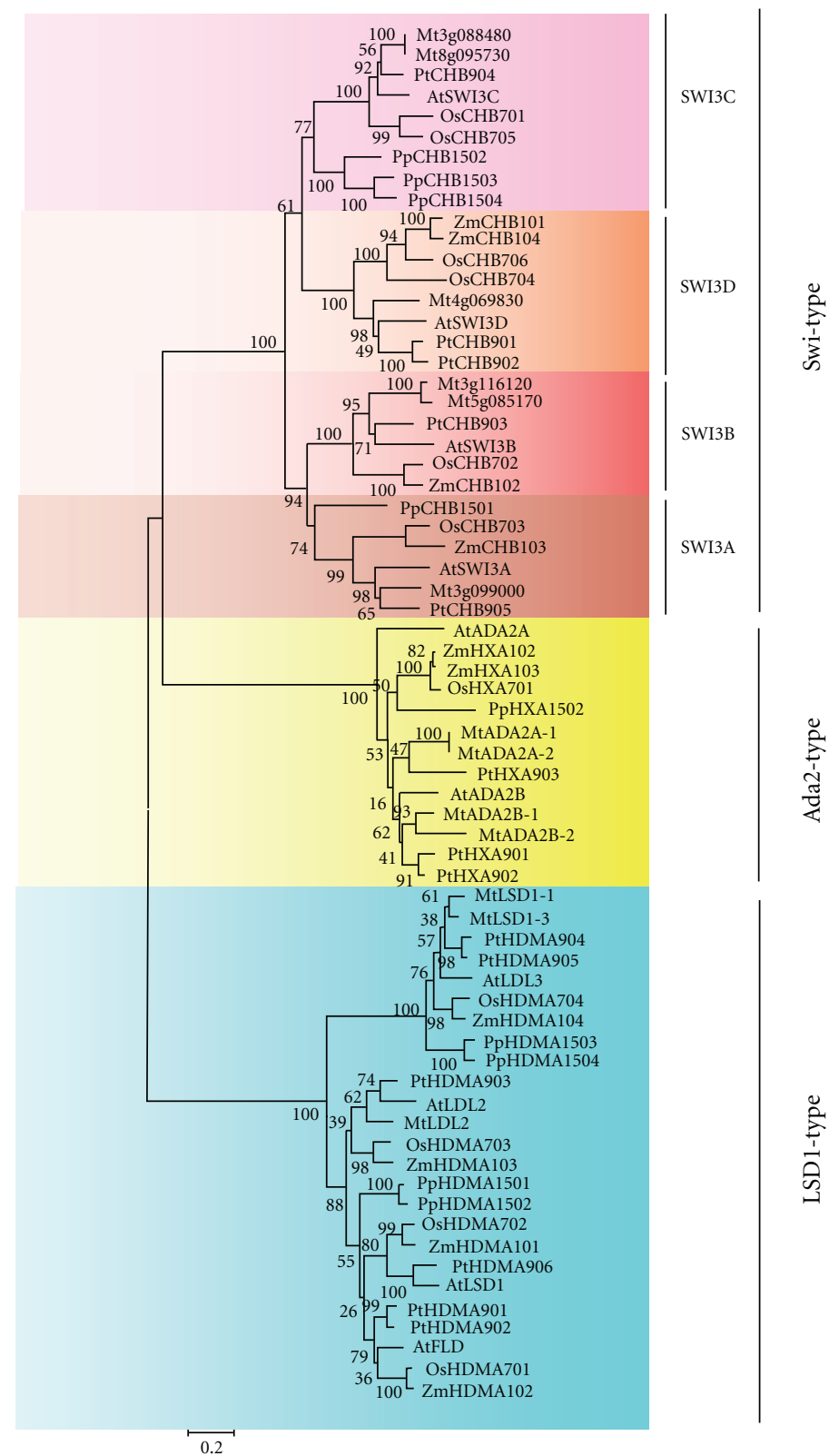

FIGURE 1: Phylogenetic analysis of SWIRM domain-containing proteins in plants. The neighbor joining phylogenetic tree constructed by MEGA4 summarizes the evolutionary relationships among the 67 members of the SWIRM domain-containing proteins from Arabidopsis thaliana (At), Medicago truncatula (Mt), Oryza sativa L. ssp. Japonica (Os), Physcomitrella patens (Pp), Populus trichocarpa (Pt), and Zea mays $(\mathrm{Zm})$.

in Oryza sativa, among which OsCHB703 belongs to the SWI3A group, OsCHB702 belongs to the SWI3B group, OsCHB701 and OsCHB705 belong to the SWI3C group, while OsCHB704 and OsCHB706 belong to the SWI3D group. Like LSD1-like subfamily in Arabidopsis thaliana, four LSD1-type SWIRM proteins were identified in Oryza sativa. It is noteworthy that there is only one Ada2-type SWIRM protein in Oryza sativa or Physcomitrella patens, while at least two or more Ada2-type SWIRM proteins in other four species.

3.2. Multiple Sequence Alignments of SWIRM Domains in Oryza sativa. To investigate the conserved amino acids of
SWIRM domains, we performed multiple sequence alignments of the 11 amino acids sequences of SWIRMs from Oryza sativa. These 11 SWIRM domains contain several conserved residues such as polar residues (YNRDTK), amphoteric residues (RQ), small residues (NDSTPASV), aliphatic residues (LIAV), and aromatic residues (YHWH) (Figure 2(a)).

Generally, the SWIRM domain forms a helix-turn-helix motif commonly found in DNA-binding proteins [28]. Based on the multiple sequence alignments, the secondarystructures of Swi3, Ada2, and LSD1 SWIRM domains in Oryza sativa were similar to those in Arabidopsis (Figures 2(b), 2(c), and 2(d)). In detail, the SWIRM domain forms 


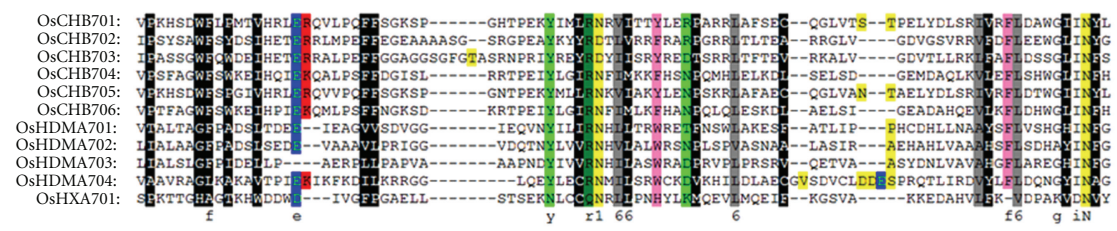

(a)

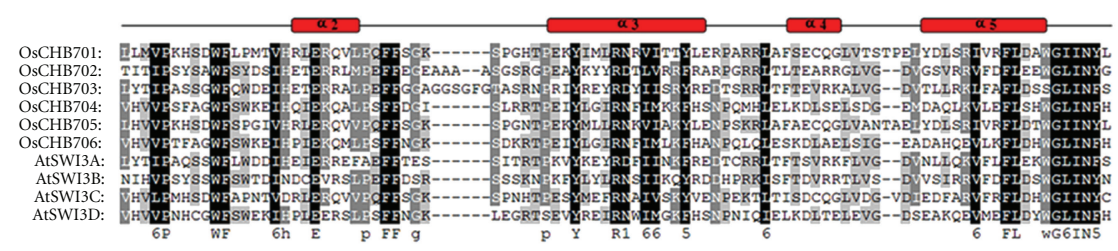

(b)

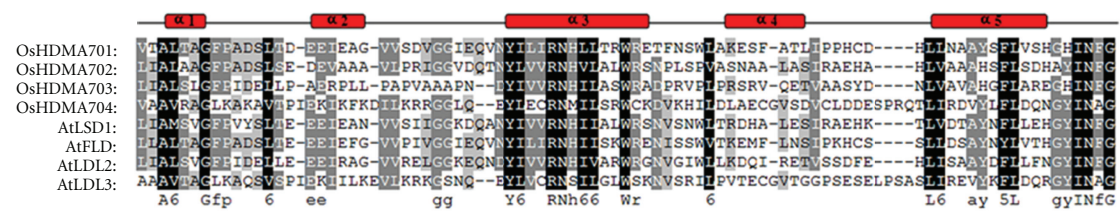

(c)

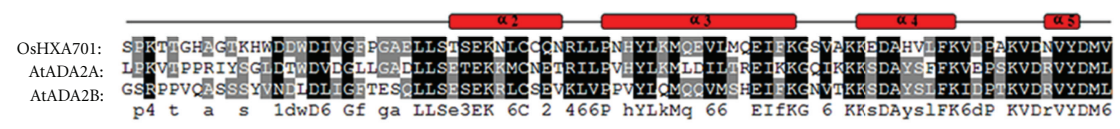

(d)

FIGURE 2: Structure-base sequence alignment of SWIRM domain subtypes. The sequence alignment was produced using Cluatal X. Resulting sequences were then adjusted manually using the GeneDoc software. The secondary structure was predicted by Jpred and PSIPRED. (a) Alignment of the SWIRM domain sequences in Oryza sativa. The coloring represents the conservation profile of amino acid residues distinguished by the following amino acid classes: hydrophobic residues are indicated by shaded black, charged residues (ED) by shaded blue, positive residues (RK) by shaded red, polar residues (YNRDTK) by shaded light green, amphoteric residues (RQ) by shaded green, small residues (NDSTPASV) by shaded yellow, aliphatic residues (LIAV) by shaded gray, and aromatic residues (YHWH) by shaded pink. (b) Alignment of the Swi3-type SWIRM domain sequences. The shaded blocks indicate several highly conserved residues by the alignment of SWIRM domain in Oryza sativa and Arabidopsis thaliana. (c) Alignment of the LSD1-type SWIRM domain sequences. The shaded blocks indicate several highly conserved residues by the alignment of SWIRM domains in Oryza sativa and Arabidopsis thaliana. (d) Alignment of the Ada2-type SWIRM domain sequences.

a helix-turn-helix-related fold. Swi3-type and Ada2-type SWIRM domains form antiparallel four helical bundles, respectively (Figures 2(b) and 2(d)). However, differing from Swi3, and Ada2-SWIRM, LSD1-SWIRM has an additional helix at its $\mathrm{N}$ terminus (Figure 2(c)).

\subsection{Analysis of Other Conserved Domains in Oryza sativa.} Based on the results from Pfam, SMART, and CDD databases, other conserved domains of SWIRM domaincontaining proteins were also identified. The results were showed in Figure 3. The structure styles of Swi and Adatype SWIRM domain-containing proteins are similar. All of them contain SWIRM and SANT domains. SANT domain, also named Myb-like DNA-binding domain, is essential for the in vivo functions in the SWI-SNF and ADA complexes. It has been implicated that SANT domain is required for an Ada2p-dependent enhancement of histone tail binding and enzymatic catalysis by Gcn5p [29]. Two members of SWI3D group, OsCHB704 and OsCHB706, have a $\mathrm{ZZ}$ zinc finger domain that could also be found in OsHXA701 (Ada2 type).
The ZZ-type zinc finger domain, named by its ability to bind two zinc ions, contains 4-6 Cys residues that participate in zinc binding (plus additional Ser/His residues). These zinc fingers are thought to be involved in protein-protein interactions [30]. In addition, OsCHB701 (SWI3C group), OsCHB702 (SWI3B group), and OsCHB703 (SWI3A group) have coiled-coil regions. All of the LSD1-type SWIRM members have an amine oxidases domain (AOD) and a SWIRM domain. Amine oxidases domains are responsible for the demethylase activity through the flavin-adeninedinucleotide- (FAD-) dependent mechanism (Figure 3(b)) [15].

3.4. Expression Pattern Analysis of OsSwirms. Since the function of a gene could be predicted by investigating its expression pattern, we analyzed the gene expression data from Genevestigator. Based on the microarray data, OsCHB705 keeps a high level of expression almost at all the developmental stages (Figure 4(a)) and in all the tissues (Figure 4(b)). OsCHB702 and OsHXA701 share the similar 
TABLE 1: SWIRM domain-containing proteins in plants.

\begin{tabular}{|c|c|c|c|c|c|c|}
\hline \multirow[b]{2}{*}{ Arabidopsis thaliana } & \multicolumn{2}{|c|}{ Swi3-type subfamily } & \multicolumn{2}{|c|}{ LSD1-type subfamily } & \multirow{2}{*}{$\begin{array}{c}\text { Ada2-type subfamily } \\
\text { AtADA2A } \\
\text { AtADA2B }\end{array}$} & \multirow{2}{*}{$\begin{array}{c}\text { Total } \\
10\end{array}$} \\
\hline & $\begin{array}{l}\text { AtSWI3A } \\
\text { AtSWI3C }\end{array}$ & $\begin{array}{l}\text { AtSWI3B } \\
\text { AtSWI3D }\end{array}$ & $\begin{array}{l}\text { AtLSD1 } \\
\text { AtLDL3 }\end{array}$ & $\begin{array}{c}\text { AtLDL2 } \\
\text { AtFLD }\end{array}$ & & \\
\hline Medicago truncatula & $\begin{array}{l}\text { Mt4g06983 } \\
\text { Mt5g08517 } \\
\text { Mt3g08848 } \\
\text { Mt3g09900 } \\
\text { Mt3g11612 } \\
\text { Mt8g09573 }\end{array}$ & $\begin{array}{l}\text { IARCC1) } \\
\text { IARCC1) } \\
\text { IARCC2) } \\
\text { IARCC2) } \\
\text { IARCC2) } \\
\text { IARCC2) } \\
\end{array}$ & $\begin{array}{l}\text { MtLDL1 } \\
\text { MtLDL2 } \\
\text { MtLDL3 }\end{array}$ & & $\begin{array}{l}\text { MtADA2A-1 } \\
\text { MtADA2A-2 } \\
\text { MtADA2B-1 } \\
\text { MtADA2B-2 }\end{array}$ & 13 \\
\hline Oryza sativa L. ssp. Japonica & $\begin{array}{l}\text { OsCHB701 } \\
\text { OsCHB703 } \\
\text { OsCHB705 } \\
\end{array}$ & $\begin{array}{l}\text { OsCHB702 } \\
\text { OsCHB704 } \\
\text { OsCHB706 }\end{array}$ & $\begin{array}{l}\text { OsHDMA701 } \\
\text { OsHDMA703 }\end{array}$ & $\begin{array}{l}\text { OsHDMA702 } \\
\text { OsHDMA704 }\end{array}$ & OsHXA701 & 11 \\
\hline Physcomitrella patens & $\begin{array}{l}\text { PpCHB1501 } \\
\text { PpCHB1503 }\end{array}$ & $\begin{array}{l}\text { PpCHB1502 } \\
\text { PpCHB1504 }\end{array}$ & $\begin{array}{l}\text { PpHDMA1501 } \\
\text { PpHDMA1503 }\end{array}$ & $\begin{array}{l}\text { PpHDMA1502 } \\
\text { PpHDMA1504 }\end{array}$ & PpHXA1502 & 9 \\
\hline Populus trichocarpa & $\begin{array}{l}\text { PtCHB901 } \\
\text { PtCHB903 } \\
\text { PtCHB905 } \\
\end{array}$ & $\begin{array}{l}\text { PtCHB902 } \\
\text { PtCHB904 }\end{array}$ & $\begin{array}{l}\text { PtHDMA901 } \\
\text { PtHDMA903 } \\
\text { PtHDMA905 } \\
\end{array}$ & $\begin{array}{l}\text { PtHDMA902 } \\
\text { PtHDMA904 } \\
\text { PtHDMA906 } \\
\end{array}$ & $\begin{array}{l}\text { PtHXA901 } \\
\text { PtHXA902 } \\
\text { PtHXA903 } \\
\end{array}$ & 14 \\
\hline Zea mays & $\begin{array}{l}\mathrm{ZmCHB} 101 \\
\mathrm{ZmCHB} 103\end{array}$ & $\begin{array}{l}\mathrm{ZmCHB} 102 \\
\mathrm{ZmCHB} 104\end{array}$ & $\begin{array}{l}\text { ZmHDMA101 } \\
\text { ZmHDMA103 }\end{array}$ & $\begin{array}{l}\text { ZmHDMA102 } \\
\text { ZmHDMA104 }\end{array}$ & $\begin{array}{l}\text { ZmHXA102 } \\
\text { ZmHXA103 }\end{array}$ & 10 \\
\hline
\end{tabular}

Alias and other names referred to the SWIRM domain-containing proteinsin Arabidopsis thaliana and Medicago truncatula were assigned by http://www .arabidopsis.org/ and http://www.jcvi.org/cgi-bin/medicago/overview.cgi. The names of SWIRM domain-containing proteins in Oryza sativa, Physcomitrella patens, Populus trichocarpa, and Zea mays are assigned by chromatin database (http://www.chromdb.org).

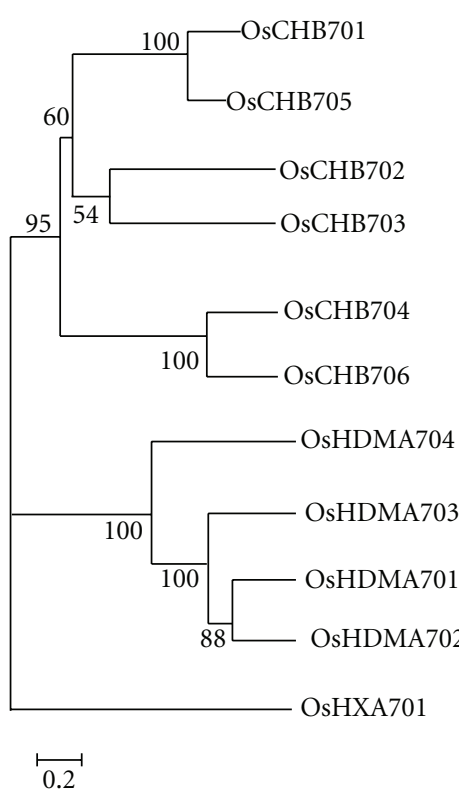

(a)

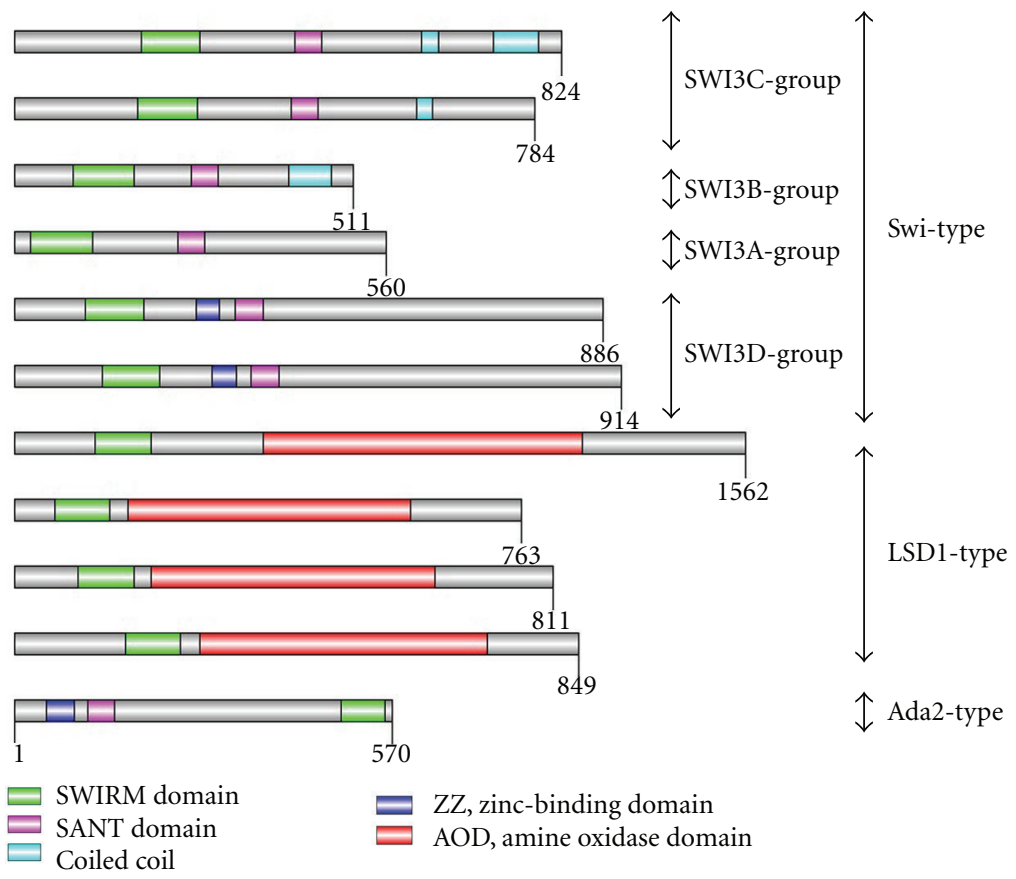

(b)

FIGURE 3: Conserved domains of SWIRM domain-containing proteins in Oryza sativa. (a) The neighbor joining phylogenetic tree constructed by MEGA4 summarizes the evolutionary relationships among the 11 members of the SWIRM domain-containing proteins from Oryza sativa. (b) The conserved domains of SWIRMs in Oryza sativa were drawn by DOG 2.0 with their corresponding amino acid lengths. 


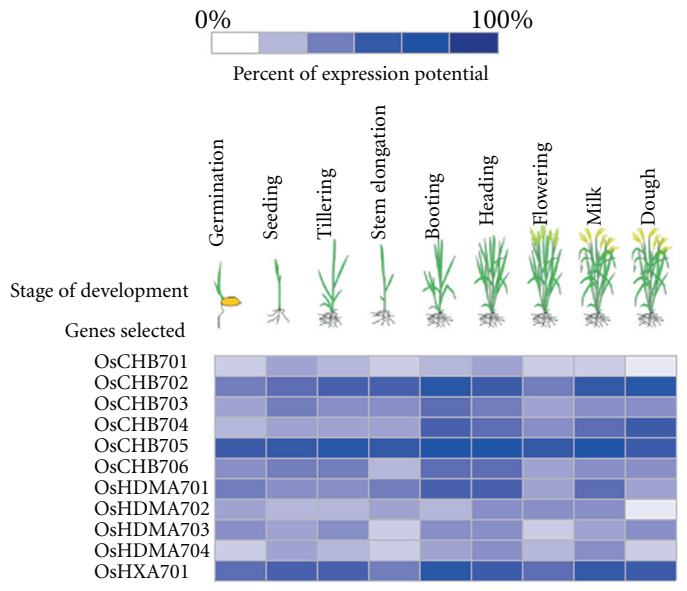

(a)

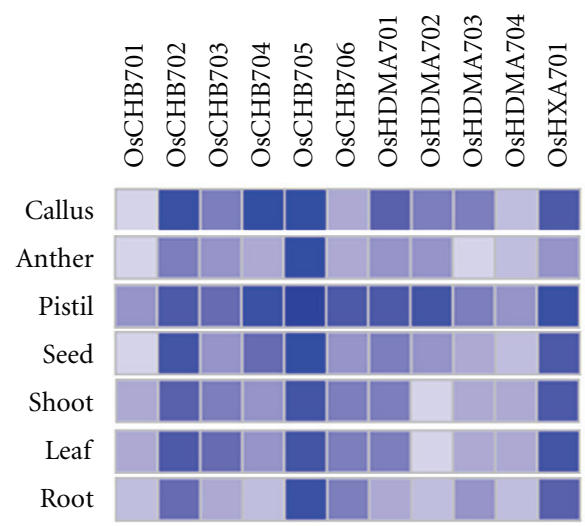

(b)

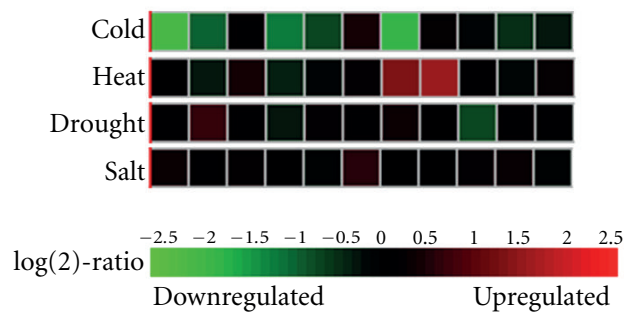

(c)

FIGURE 4: Expression patterns of genes encoding SWIRM family proteins in Oryza sativa based on Genevestigator. (a)-(b) Expression patterns of SWIRM genes in different developmental stages and different anatomical parts. The darkest blue color represents the maximum level of expression for a given gene across all measurements available in the database for this gene. This means that color intensities can only be compared between elements from the same gene but not with those from other genes. The expression potential of a gene is a robust measure for the maximum of the expression level for this gene. The expression potential is defined as the average of the top $1 \%$ signal values across all samples for a given probe set in a given platform. (c) Expression patterns of SWIRM genes induced by abiotic stress. Green-red color coding bar represents ratios of treatment versus control values. Gray is used to indicate that both treatment and control are in the background range and that therefore the ratio is not robust. The background threshold $B(x)$ is a probe set specific expression level. It is defined as $B(x) \leq 50 \%$ quantile of all signals measured of probe set $x$ having "absent" calls ( $P$ value $>0.05)$ according to MAS5.

expression profile in developmental process and their highest expression level can be observed at booting, milk, and dough stages (Figure 4(a)). However, OsCHB702 expresses highest in callus, while OsHXA701 expresses highest in pistil (Figure 4(b)). In addition, OsCHB701, OsHDMA702, OsHDMA703, and OsHDMA704 show relatively low expression in all developmental stages (Figure 4(a)) and tissues (Figure 4(b)). Interestingly, compared with the expression level in other tissues, most OsSWIRMs show high expression in the pistil (Figure 4(b)).

The effects of abiotic stresses including cold, heat, drought, and salt on the expression of the 11 OsSWIRMs were also investigated. The expression of OsHDMA701 was significantly down-regulated by cold but up-regulated by heat (Figure 4(c)), implying that OsHDMA701 was involved in the response to the change in temperature. In addition, the

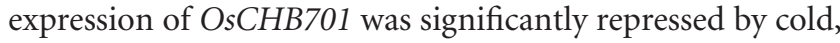
while the expression of OsHDMA702 was enhanced by heat (Figure 4(c)). However, the expression of other OsSWIRMs was not affected by these abiotic stresses.

\section{Conclusion and Discussion}

67 SWIRM domain-containing proteins from six plant species were identified and could be divided into three distinct types: Swi-type, LSD1-type, and Ada2-type (Figure 1). No MYSM-type SWIRM domain-containing proteins were found in plants (Figure 1), although they were widely observed in animals $[13,31]$, indicating that the SWIRM domain-containing proteins may evolve differently between plants and animals. The further analysis suggested that the Swi3-type and Ada2-type SWIRM proteins may diverged after the appearance of the LSD1-type SWIRM (Figure 1). Consistent with the previous reports $[12,32]$, all of these SWIRM structures in plant form a hHTH-related motif commonly found in DNA-binding proteins and tend to form a long central $\alpha$-helix surrounded by several short helices (Figure 2). In addition, besides the conserved SWIRM domain, each type of SWIRM domain-containing proteins harbored their own distinct motifs, such as SANT, ZZ, and AOD (Figure 3), implying the distinction in function among these proteins. 
There is increasing evidence showing that SWIRM proteins in plants play a crucial role in a range of developmental processes and in responses to abiotic stresses [33]. The expression of the SWI3C group OsCHB701 and $\mathrm{OsCHB705}$ was not changed appreciably in different developmental stages, while OSCHB702 and OsHDMA701 were up-regulated during the booting and heading stage (Figure 4(a)). On the other hand, the expression of Ada2type SWIRM OsHXA701 was the lowest in anther, but increased in booting stage and milk stage (Figure 4(a)). The OsCHB702 homolog AtSWI3B was found to interact with FCA, a regulator of flowering time in Arabidopsis [19]. In addition, OsHDMA701 homolog AtFLD can interact with CO (CONSTANS) to affect both flowering time and floral initiation in Arabidopsis [21, 22]. It remains to be determined whether OSCHB702 and OsHDMA701 are also involved in regulating flower time in Oryza sativa.

OsCHB702 is highly expressed in callus and seeds. The atswi3b mutations resulted in the early embryo lethality [17]. It is possible that $\mathrm{OsCHB702}$ may also be involved in embryonic and seed development in Oryza sativa. Most OsSWIRMs are highly expressed in the pistil. In Arabidopsis, mutations in AtSWI3C and AtSWI3D cause aberrant stamen development and abnormal carpel development $[17,20]$. These observations suggest that SWI3-type proteins may play a significant role in floral development. Furthermore, OsCHB701, OsCHB705, and OsHDMA701 can be down-regulated by cold stress, while OsHDMA701 and OsHDMA702 were significantly induced by the heat stress, suggesting a role of these OsSWIRMs in abiotic stress response. Our study provides insights into the evolution and function of the plant SWIRM domain proteins. Further studies are required to use functional genetics tools to elucidate clearly the functions of SWIRM domain-containing proteins in different plant species.

\section{Authors' Contribution}

The authors Y. Gao and S. Yang contributed equally to the paper.

\section{Acknowledgments}

This work was supported by the National Basic Research Program of China (973 program no. 2012CB910900) and the Grants from Natural Science Foundation of China (no. 90919038, no. 30971564, and no. 31128001).

\section{References}

[1] A. E. Ehrenhofer-Murray, "Chromatin dynamics at DNA replication, transcription and repair," European Journal of Biochemistry, vol. 271, no. 12, pp. 2335-2349, 2004.

[2] C. Wu, "Chromatin remodeling and the control of gene expression," The Journal of Biological Chemistry, vol. 272, no. 45, pp. 28171-28174, 1997.

[3] K. Luger, "Structure and dynamic behavior of nucleosomes," Current Opinion in Genetics and Development, vol. 13, no. 2, pp. 127-135, 2003.
[4] A. I. Lamond and W. C. Earnshaw, "Structure and function in the nucleus," Science, vol. 280, no. 5363, pp. 547-553, 1998.

[5] C. Muchardt and M. Yaniv, "ATP-dependent chromatin remodelling: SWI/SNF and Co. are on the job," Journal of Molecular Biology, vol. 293, no. 2, pp. 187-198, 1999.

[6] L. Zeng and M. M. Zhou, "Bromodomain: an acetyl-lysine binding domain,” FEBS Letters, vol. 513, no. 1, pp. 124-128, 2002.

[7] S. Mujtaba, L. Zeng, and M. M. Zhou, "Structure and acetyllysine recognition of the bromodomain," Oncogene, vol. 26, no. 37, pp. 5521-5527, 2007.

[8] A. Akhtar, D. Zink, and P. B. Becker, "Chromodomains are protein-RNA interaction modules," Nature, vol. 407, no. 6802, pp. 405-409, 2000.

[9] I. Holdermann, N. H. Meyer, A. Round, K. Wild, M. Sattler, and I. Sinning, "Chromodomains read the arginine code of post-translational targeting," Nature Structural and Molecular Biology, vol. 19, no. 2, pp. 260-263, 2012.

[10] R. Aasland, A. F. Stewart, and T. Gibson, "The SANT domain: a putative DNA-binding domain in the SWI-SNF and ADA complexes, the transcriptional co-repressor N-CoR and TFIIIB," Trends in Biochemical Sciences, vol. 21, no. 3, pp. 87$88,1996$.

[11] L. A. Boyer, M. R. Langer, K. A. Crowley, S. Tan, J. M. Denu, and C. L. Peterson, "Essential role for the SANT domain in the functioning of multiple chromatin remodeling enzymes," Molecular Cell, vol. 10, no. 4, pp. 935-942, 2002.

[12] L. Aravind and L. M. Iyer, "The SWIRM domain: a conserved module found in chromosomal proteins points to novel chromatin-modifying activities," Genome Biology, vol. 3, no. 8, 2002.

[13] M. Yoneyama, N. Tochio, T. Umehara et al., "Structural and functional differences of SWIRM domain subtypes," Journal of Molecular Biology, vol. 369, no. 1, pp. 222-238, 2007.

[14] N. Tochio, T. Umehara, S. Koshiba et al., "Solution structure of the SWIRM domain of human histone demethylase LSD1," Structure, vol. 14, no. 3, pp. 457-468, 2006.

[15] Y. Shi, F. Lan, C. Matson et al., "Histone demethylation mediated by the nuclear amine oxidase homolog LSD1," Cell, vol. 119, no. 7, pp. 941-953, 2004.

[16] D. E. Sterner, X. Wang, M. H. Bloom, G. M. Simon, and S. L. Berger, "The SANT domain of Ada2 is required for normal acetylation of histones by the yeast SAGA complex," The Journal of Biological Chemistry, vol. 277, no. 10, pp. 8178 8186, 2002.

[17] T. J. Sarnowski, G. Rios, J. Jásik et al., "SWI3 subunits of putative SWI/SNF chromatin-remodeling complexes play distinct roles during Arabidopsis development," Plant Cell, vol. 17, no. 9, pp. 2454-2472, 2005.

[18] A. Saez, A. Rodrigues, J. Santiago, S. Rubio, and P. L. Rodriguez, "HAB1-SWI3B interaction reveals a link between abscisic acid signaling and putative SWI/SNF chromatinremodeling complexes in Arabidopsis," Plant Cell, vol. 20, no. 11, pp. 2972-2988, 2008.

[19] T. J. Sarnowski, S. Świezewski, K. Pawlikowska, S. Kaczanowski, and A. Jerzmanowski, "AtSWI3B, an Arabidopsis homolog of SWI3, a core subunit of yeast Swi/Snf chromatin remodeling complex, interacts with FCA, a regulator of flowering time," Nucleic Acids Research, vol. 30, no. 15, pp. 3412-3421, 2002.

[20] R. Archacki, T. J. Sarnowski, J. Halibart-Puzio et al., "Genetic analysis of functional redundancy of BRM ATPase and ATSWI3C subunits of Arabidopsis SWI/SNF chromatin 
remodelling complexes," Planta, vol. 229, no. 6, pp. 12811292, 2009.

[21] M. L. Chou and C. H. Yang, "FLD interacts with genes that affect different developmental phase transitions to regulate Arabidopsis shoot development," Plant Journal, vol. 15, no. 2, pp. 231-242, 1998.

[22] Y. He, S. D. Michaels, and R. M. Amasino, "Regulation of flowering time by histone acetylation in Arabidopsis," Science, vol. 302, no. 5651, pp. 1751-1754, 2003.

[23] S. F. Altschul, W. Gish, W. Miller, E. W. Myers, and D. J. Lipman, "Basic local alignment search tool," Journal of Molecular Biology, vol. 215, no. 3, pp. 403-410, 1990.

[24] K. Tamura, J. Dudley, M. Nei, and S. Kumar, "MEGA4: molecular Evolutionary Genetics Analysis (MEGA) software version 4.0," Molecular Biology and Evolution, vol. 24, no. 8, pp. 1596-1599, 2007.

[25] J. D. Thompson, T. J. Gibson, F. Plewniak, F. Jeanmougin, and D. G. Higgins, "The CLUSTAL X windows interface: flexible strategies for multiple sequence alignment aided by quality analysis tools," Nucleic Acids Research, vol. 25, no. 24, pp. 4876-4882, 1997.

[26] K. B. Nicholas, H. B. J. Nicholas Jr., and D. W. Deerfield II, "GeneDoc: analysis and visualization of genetic variation," EMBnet.News, vol. 4, pp. 1-4, 1997.

[27] J. Ren, L. Wen, X. Gao, C. Jin, Y. Xue, and X. Yao, "DOG 1.0: illustrator of protein domain structures," Cell Research, vol. 19, no. 2, pp. 271-273, 2009.

[28] R. Marmorstein, G. P. Da, J. Lenkart, K. Zhao, R. Shiekhattar, and B. R. Cairns, "Structure and function of the SWIRM domain, a conserved protein module found in chromatin regulatory complexes," The Faseb, vol. 20, no. 4, pp. A34-A35, 2006.

[29] A. M. Gamper, J. Kim, and R. G. Roeder, "The STAGA subunit ADA2b Is an important regulator of human GCN5 catalysis," Molecular and Cellular Biology, vol. 29, no. 1, pp. 266-280, 2009.

[30] G. B. Legge, M. A. Martinez-Yamout, D. M. Hambly et al., "ZZ domain of CBP: an unusual zinc finger fold in a protein interaction module," Journal of Molecular Biology, vol. 343, no. 4, pp. 1081-1093, 2004.

[31] J. W. B. Hershey, K. Asano, T. Naranda, H. P. Vornlocher, P. Hanachi, and W. C. Merrick, "Conservation and diversity in the structure of translation initiation factor eIF3 from humans and yeast," Biochimie, vol. 78, no. 11-12, pp. 903-907, 1996.

[32] G. P. Da, J. Lenkart, K. Zhao, R. Shiekhattar, B. R. Cairns, and R. Marmorstein, "Structure and function of the SWIRM domain, a conserved protein module found in chromatin regulatory complexes," Proceedings of the National Academy of Sciences of the United States of America, vol. 103, no. 7, pp. 2057-2062, 2006.

[33] A. Jerzmanowski, "SWI/SNF chromatin remodeling and linker histones in plants," Biochimica et Biophysica Acta, vol. 1769, no. 5-6, pp. 330-345, 2007. 

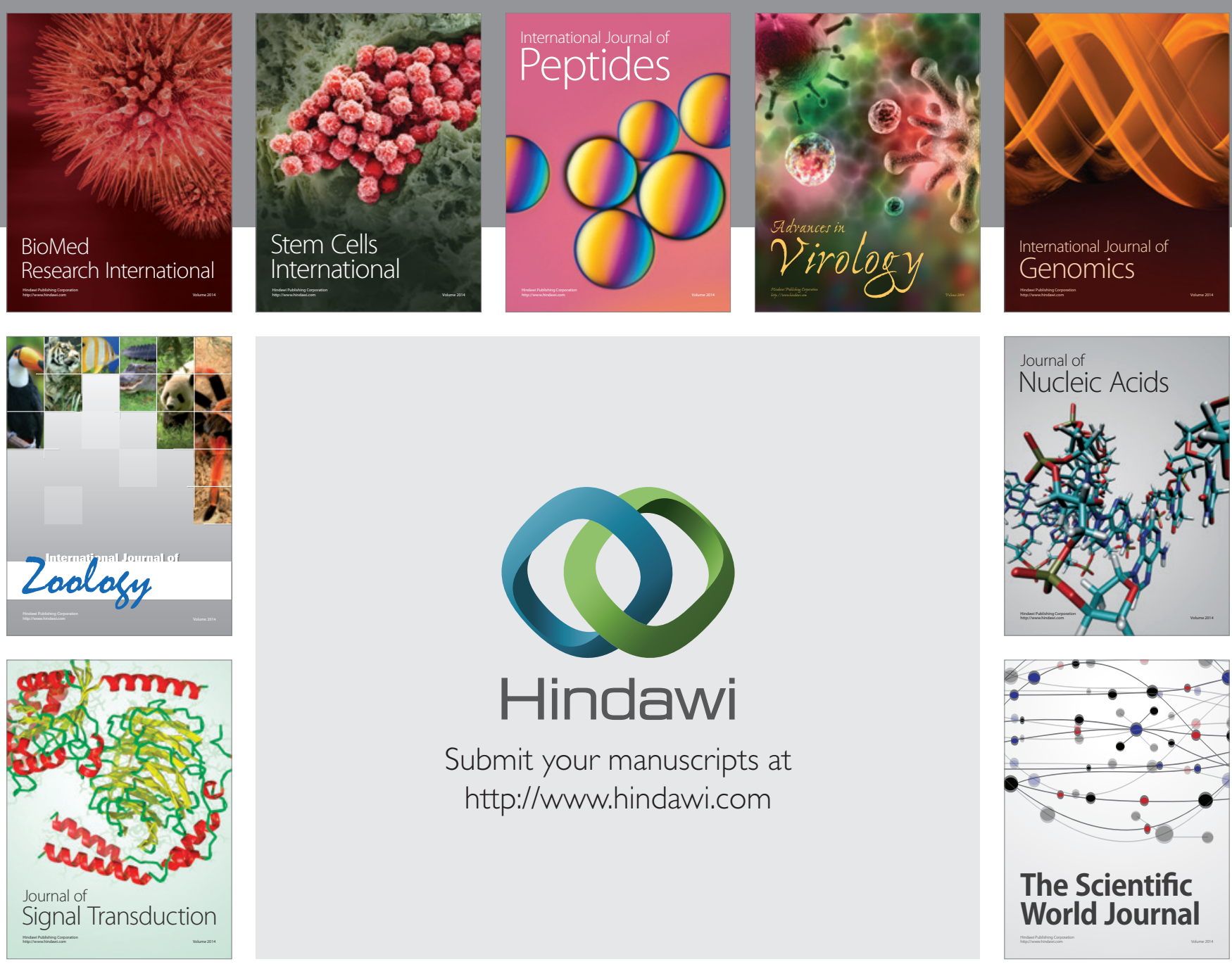

Submit your manuscripts at

http://www.hindawi.com
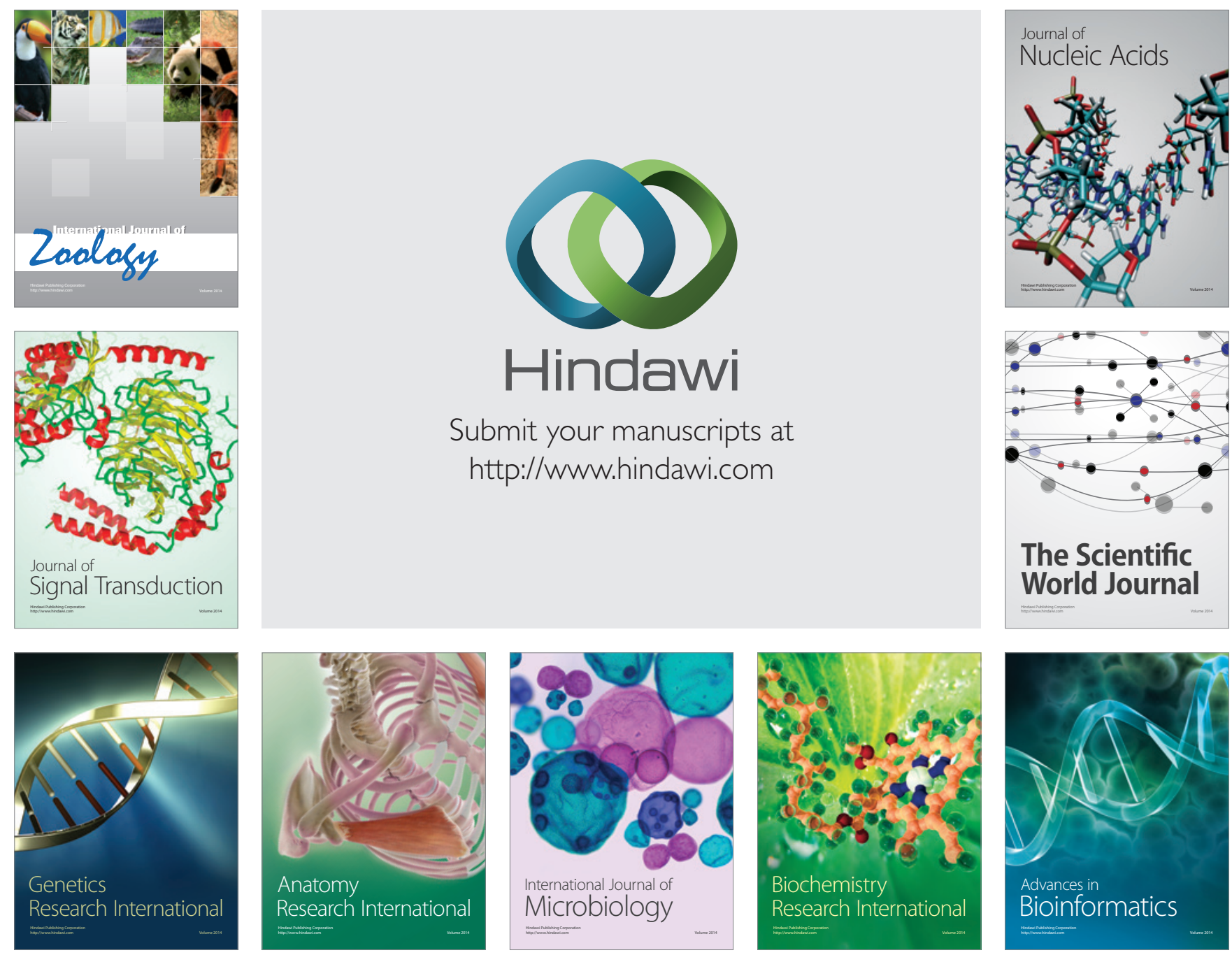

The Scientific World Journal
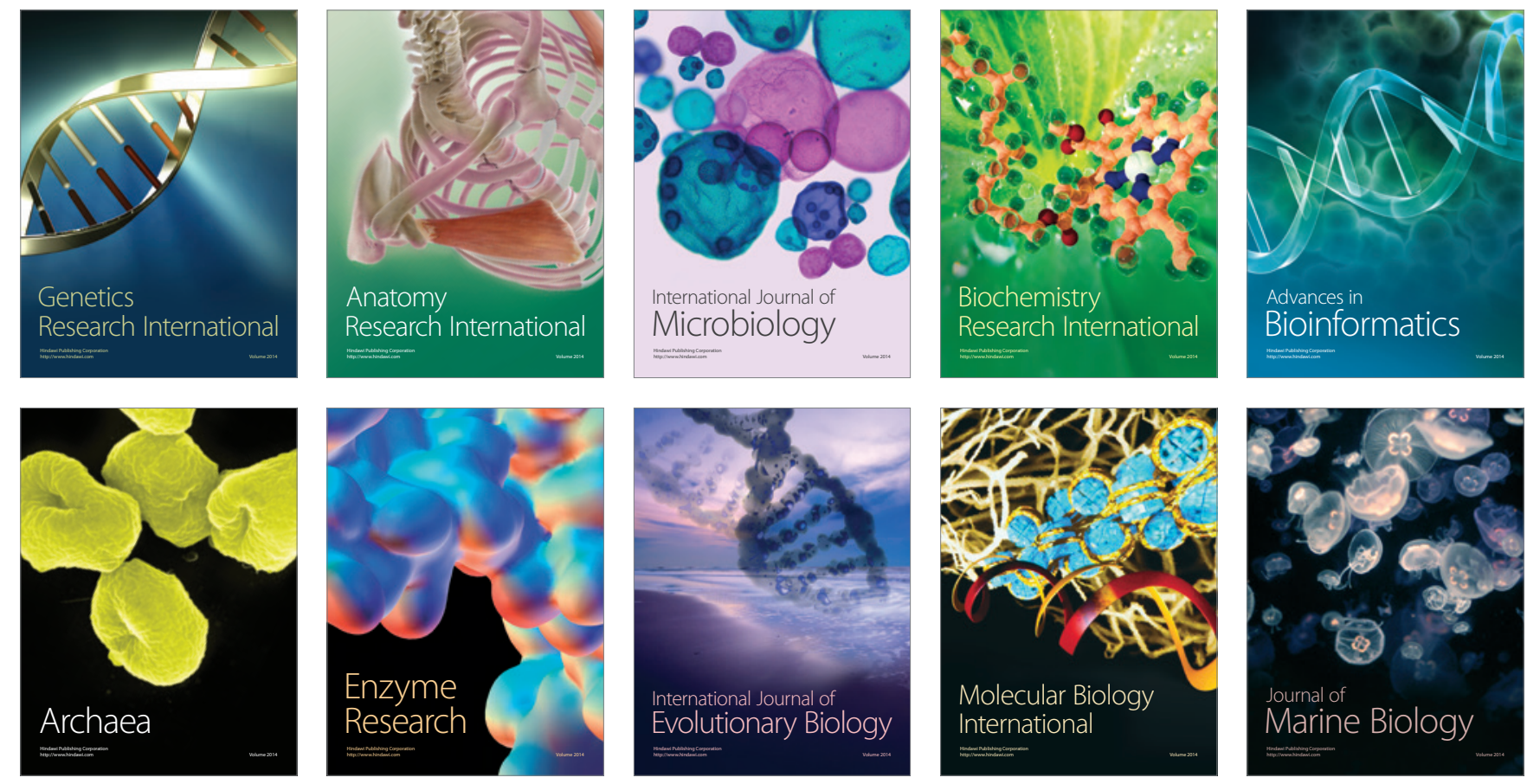